\title{
Consumo alimentar de idosos residentes na zona rural do município de Caxias do Sul, RS
}

\author{
Food consumption of elderly residents in the rural area of \\ Caxias do Sul municipal, RS
}

\author{
Gabriela Schmitt Marchesi ${ }^{1}$, Simara Rufatto Conde
}

${ }^{1}$ Curso de Bacharelado em Nutrição, Faculdade e Escola de Educação Profissional Nossa Senhora de Fátima (FEEPNSF) - Caxias do Sul (RS), Brasil.

DOI: http://dx.doi.org/10.7322/abcshs.v43i3.1089

\begin{abstract}
RESUMO
Introdução: O Brasil apresenta um grande índice de envelhecimento populacional, característica que envolve grandes desafios, sendo um deles o processo de alimentação. Idosos que residem na zona rural possuem maior oferta de alimentos in natura, fato que ocorre pelo cultivo dos mesmos em suas residências. Objetivo: Avaliar o perfil alimentar da população idosa residente na zona rural do Município de Caxias do Sul. Métodos: Estudo transversal cuja amostra se deu por conveniência, composta por 16 idosos de ambos os gêneros que participaram do projeto Conviver. Foi avaliado o estado mental dos participantes através do Mini Exame do Estado Mental e a ingestão alimentar por meio do recordatório alimentar de 24 horas com relato de três dias. Os dados foram analisados estatisticamente através do Teste t de Student e do Teste de Mann-Whitney através do software SPSS versão 22. Resultados: $O$ consumo de processados e ultraprocessados pelos idosos representou 30,28\% do valor energético total. Os idosos apresentaram consumo acima do estabelecido pela DRI para carboidratos e abaixo para lipídios, cálcio, fibras, vitaminas A, B6 e B12. A ingestão de proteína, sódio, ferro e vitamina C estavam dentro das recomendações. Conclusão: Os idosos participantes do estudo apresentam uma alimentação rica em carboidratos, porém com deficiências de micronutrientes.
\end{abstract}

Palavras-chave: saúde do idoso; nutrição do idoso; fenômenos fisiológicos da nutrição do idoso; alimentos, dieta e nutrição.

\begin{abstract}
Introduction: Brazil presents a high index of population aging, a characteristic that involves great challenges, one of them being the feeding process. Older people residing in the rural area have a greater supply of in natura food, a fact that occurs through the cultivation of the same in their residences. Objective: To evaluate the food profile of the elderly population residing in the rural area of the Municipality of Caxias do Sul. Methods: A cross-sectional study was carried out by 16 elderly people of both sexes who participated in the Conviver project. The mental state of the participants was evaluated through the Mini Mental State Examination and food intake through a 24hour food recall with a three-day report. Data were analyzed statistically through the Student's t-Test and the Mann-Whitney Test using SPSS software version 22. Results: The consumption of processed and ultraprocessed by the elderly represented $30.28 \%$ of the total energy value. The elderly presented consumption above the established by DRI for carbohydrates and below for lipids, calcium, fiber, vitamins A, B6 and B12. The intake of protein, sodium, iron and vitamin $\mathrm{C}$ were within the recommendations. Conclusion: The elderly participants of the study presented a diet rich in carbohydrates, but with deficiencies of micronutrients.
\end{abstract}

Keywords: health of the elderly; elderly nutrition; elder nutritional physiological phenomena; diet, food, and nutrition.

Recebido em: 04/12/2017

Revisado em: 06/06/2018

Aprovado em: 07/08/2018

Autor para correspondência: Simara Rufatto Conde - Associação Cultural e Científica Virvi Ramos - Faculdade Nossa Senhora de Fátima - Rua Alexandre Fleming, 454 - Madureira - CEP: 95041-520 - Caxias do Sul (RS), Brasil - Email: simararufatto@terra.com.br

Conflito de interesses: nada a declarar. 


\section{INTRODUÇÃO}

O Brasil apresenta uma alta taxa de envelhecimento populacional quando comparada com a natalidade, fato que gera grandes desafios em âmbitos sociais, políticos e econômicos ${ }^{1}$. Diante do acelerado envelhecimento da população brasileira, torna-se fundamental o planejamento e o desenvolvimento de ações direcionadas a promoção da saúde dos idosos, incluindo a alimentação saudável e adequada ${ }^{2}$.

Diversos fatores fisiológicos, psicológicos e sociais, incluindo problemas de mastigação, deglutição, perda de apetite, isolamento social e limitações causadas por doenças crônicas não transmissíveis podem provocar mudanças negativas no padrão alimentar dos idosos ${ }^{3,4}$. Conforme estudo realizado por Venturini et al. ${ }^{5}$ existem importantes deficiências nutricionais na população idosa, tais como deficiências de vitaminas e minerais em razão de uma preferência por alimentos ricos em carboidratos.

Por mais que os idosos que residem na zona rural optem mais por alimentos in natura, como arroz, feijão, batata-doce, mandioca, farinha de mandioca, frutas e peixes, por poderem cultivar diferentes qualidades de frutas e verduras nos seus quintais e hortas, consumindo tais alimentos ${ }^{2}$ a ingestão de vitaminas e minerais da população idosa encontra-se abaixo da recomendação, achado que preconiza a importância de políticas de alimentação e nutrição que estimulem o consumo de alimentos saudáveis fontes de micronutrientes ${ }^{6}$.

Outra explicação é o hábito dos brasileiros em consumirem alimentos processados, fator que está diretamente relacionado com o excesso de peso e os distúrbios metabólicos por sua vez mais frequente na população ${ }^{7,8}$.

Diante deste contexto, o presente estudo teve como objetivo avaliar o perfil alimentar da população idosa residente na zona rural do Município de Caxias do Sul, participante do projeto Conviver de Vila Seca.

\section{MÉTODOS}

Trata-se de uma pesquisa transversal de caráter quantitativo, a qual avaliou consumo alimentar dos idosos participantes do projeto Conviver, com o objetivo de promover a recreação e socialização entre os idosos, da zona rural do Município de Caxias do Sul, em Vila Seca, no estado do Rio Grande do Sul. A amostra foi por conveniência e composta por 16 idosos. A coleta de dados ocorreu entre os meses de Agosto à Outubro de 2017.

Os critérios de inclusão foram: idade igual ou superior a 60 anos, ser residentes da zona rural do município de Caxias do Sul, em Vila Seca, participar do projeto Convier e assinar o Termo de Consentimento Livre e Esclarecido (TCLE). Como critérios de exclusão foram considerados: idosos portadores de alguma doença crônica não transmissível que comprometesse a adequada resposta aos questionamentos, idosos que não foram encontrados após três encontros ou que haviam mudado de endereço.

Os idosos foram informados previamente a respeito da pesquisa e de seus objetivos. A coleta de dados foi realizada pela pesquisadora principal do estudo, por meio de uma entrevista individualizada, em uma sala reservada. Aplicou-se o Mini Exame de Estado Mental (MEEM) para avaliação do idoso quanto a sua capacidade de responder o Recordatório Alimentar de 24 horas (R24). Para os idosos aptos foi aplicado o R24 com relato de três dias não consecutivos, sendo dois dias de semana e um de final de semana, todos respondidos em uma única entrevista.

Foram avaliados através dos recordatórios de 24 horas a ingestão de alimentos processados e ultraprocessados, os macronutrientes (carboidrato, proteína, lipídeos e fibras) e os micronutrientes (sódio, cálcio, ferro, vitamina A, B6, B12, vitamina C). Os macronutrientes e micronutrientes foram comparados com a Dietary Recomended Intakes (DRI) com base na EAR. Para classificação quanto aos alimentos processados e ultraprocessados, foi utilizado como base o Guia Alimentar para a População Brasileira 2014.

O software utilizado para o cálculo do consumo alimentar foi o AVANUTRI 4.0, e foi calculada a média dos três dias do recordatório de $24 \mathrm{hs}$.

Os dados foram analisados estatisticamente por meio do teste $\mathrm{t}$ de Student para amostras independentes com distribuição normal, e do Teste de Mann-Whitney para as variáveis que não apresentaram distribuição normal. Adotou-se um nível de significância de 5\% para os testes. Todas as análises estatísticas foram realizadas no software SPSS versão 22.

O estudo foi aprovado pelo Comitê de Ética em Pesquisa da Associação Cultural e Científica Virvi Ramos pelo protocolo número 2.196.715.

\section{RESULTADOS}

A amostra foi composta por 16 indivíduos com idade média de $68,3 \pm 6,18$ anos, sendo $87 \%$ (14) do sexo feminino e $13 \%$ (2) do sexo masculino.

O consumo de alimentos processados e ultraprocessados pelos idosos representaram 30,28\% do Valor Energético Total (VET) total. Quando comparada a faixa etária com o consumo de alimentos processados e ultraprocessados constatou-se que não houve diferença significativa, embora os idosos acima de 70 anos tenham apresentado maior consumo destes alimentos ( $p$ 0,398) Teste-T de Student (Tabela 1).

A média de ingestão de carboidratos foi de $62,25 \pm 6,82 \%$, a de proteínas foi de 10,56 $\pm 6,54 \%$, de lipídeos de 10,56 $\pm 6,84 \%$ e de fibras 17,18 $\pm 9,66 \mathrm{~g}$. Quando comparada a ingestão de macronutrientes com a faixa etária, observou-se que não houve diferença significativa $(p>0,05)$. Quando comparados o consumo

Tabela 1: Comparação do consumo de processados e ultraprocessados pelos idosos entre 60-70 anos e maiores de 70 anos. Caxias do Sul, RS, 2017.

\begin{tabular}{|l|c|c|c|c|}
\hline & $\begin{array}{c}\text { Total } \\
\text { Média } \pm \text { DP }\end{array}$ & $\begin{array}{c}60-70 \text { anos } \\
\text { Média } \pm \text { DP }\end{array}$ & $\begin{array}{c}>70 \text { anos } \\
\text { Média } \pm D P\end{array}$ & P \\
\hline$\%$ VET & $30,28 \pm 19,17$ & $25,88 \pm 15,42$ & $37,63 \pm 23,91$ & 0,248 \\
\hline VET: Valor Energético Total.
\end{tabular}


dos micronutrientes: sódio, cálcio, ferro, vitamina A, B6, B12 e C com a faixa etária, não apresentou diferença significativa $(\mathrm{p}>0,05)$ Teste-T de Student (Tabela 2).

Em comparação com a DRI com base na EAR, o consumo médio do macronutriente carboidrato teve valores significativamente superiores ao recomendado $(\mathrm{p}<0,001)$. Já o consumo de proteínas estava dentro das recomendações. Os lipídios, fibras, cálcio, vitamina A, B6 e B12 apresentaram consumo médio abaixo do recomendado. Em relação aos micronutrientes, a ingestão da vitamina $\mathrm{C}$, sódio e ferro estavam de acordo com as recomendações estabelecidas, Teste de Mann-Whitney (Tabela 3).

\section{DISCUSSÃo}

No presente estudo, constatou-se que o consumo de alimentos processados e ultraprocessados pelos idosos representaram 30,28\% do valor energético total da dieta, sendo os principais deles as massas, pães, geleias, queijos e salames, resultado que, conforme pesquisa de Assumpção et al. ${ }^{9}$ colaboram para a baixa qualidade da alimentação de idosos em relação àqueles que tem preferência por alimentos in natura, visto que o consumo dessa classe de alimentos está diretamente associada ao alto teor de gordura saturadas, trans e açúcares livres e negativamente associada ao consumo proteico e de fibras, colaborando para o aumento do risco de obesidade, diabetes, doenças cardiovasculares e alguns tipos de câncer ${ }^{10}$.

Quando comparado o consumo de carboidratos com as recomendações estabelecidas pela DRI, encontrou-se valores significativamente acima do recomendado, diferindo dos resultados encontrados por Ventiruni et al..$^{5}$ os quais avaliaram o consumo de nutrientes de 427 idosos residentes de Porto Alegre, e constataram que o consumo deste macronutriente aumentava com o avanço da idade. O elevado consumo de carboidratos, principalmente os de alto índice glicêmico, está diretamente relacionado com o acúmulo de gordura corporal, sendo que um moderado consumo tem impacto na redução do surgimento de doenças cardiovasculares ${ }^{11}$.

No presente estudo a ingestão proteica, ficou significativamente dentro do recomendado pela DRI, contrário ao estudo realizado com 35 idosos da cidade de Paracatu, Minas Gerais, no qual a ingestão de proteínas foi maior que as recomendações ( $\mathrm{p}>0,05)$, no entanto o predomínio foi de proteína de alto valor biológico ${ }^{12}$. O consumo adequado de proteínas de qualidade por idosos, tem papel importante no aumento da massa muscular e para a sua manutenção a longo prazo, auxiliando assim, no retardo do surgimento da sarcopenia e suas complicações ${ }^{13}$.

Neste estudo foi constatada ingestão de lipídeos abaixo das recomendações, semelhante ao que verificaram Kucera et al..$^{14} \mathrm{em}$ estudo realizado com 90 idosos participantes de um projeto na Universidade de Caxias do Sul (UCS), em que o consumo de lipídeos de $76,6 \%$ dos idosos ficou abaixo do recomendado. $\mathrm{O}$ alto consumo de lipídios na população idosa brasileira está associado ao aumento do risco de doenças crônicas ${ }^{15}$, fato que está relacionado com a transição nutricional e com o alto consumo de ultraprocessados constatados na população estudada, o que proporciona crescente oferta de alimentos industrializados ricos em gorduras, açúcares e sódio ${ }^{16}$.

O consumo de fibras alimentares ficou significativamente abaixo da recomendação, apesar da maioria dos idosos consumirem frutas e verduras diariamente, porém não em quantidade suficiente, diferentemente dos resultados encontrados

Tabela 3: Comparação dos nutrientes consumidos com as recomendações da DRI. Caxias do Sul, RS, 2017.

\begin{tabular}{|l|c|c|c|}
\hline Macronutrientes & $\begin{array}{c}\text { Consumo total } \\
\text { Média } \pm \text { DP }\end{array}$ & DRI & p \\
\hline Carboidratos (\%) & $62,25 \pm 6,82$ & $50-60 \%$ & 0,834 \\
\hline Proteínas (\%) & $10,56 \pm 6,54$ & $18-20 \%$ & 0,001 \\
\hline Lipídios (\%) & $10,56 \pm 6,84$ & $15-30 \%$ & 0,408 \\
\hline Fibras (g) & $17,18 \pm 9,66$ & $30 \mathrm{~g}$ & 0,001 \\
\hline Micronutrientes & $\begin{array}{c}\text { Consumo total } \\
\text { Média } \pm \text { DP }\end{array}$ & DRI & $\mathbf{p}$ \\
\hline Sódio $(\mathrm{mg})$ & $1375,73 \pm 839,83$ & $1,3 \mathrm{~g}$ & 0,125 \\
\hline Cálcio $(\mathrm{mg})$ & $353,44 \pm 170,20$ & $1200 \mathrm{mg}$ & 0,379 \\
\hline Ferro $(\mathrm{mg})$ & $10,76 \pm 4,81$ & $8 \mathrm{mg}$ & 0,108 \\
\hline Vitamina A (mcg) & $394,90 \pm 618,24$ & $900 \mu \mathrm{g}$ & 0,001 \\
\hline Vitamina B6 (mg) & $0,89 \pm 1,06$ & $1,7 \mathrm{mg}$ & 0,001 \\
\hline Vitamina B12 $(\mathrm{mcg})$ & $1,18 \pm 1,32$ & $2,4 \mu \mathrm{g}$ & 0,001 \\
\hline Vitamina C $(\mathrm{mg})$ & $118,85 \pm 63,51$ & $90 \mathrm{mg}$ & 0,603 \\
\hline
\end{tabular}

DRI: Dietary Recomended Intakes.

Tabela 2: Ingestão média de macro e micronutrientes por idosos ( $\geq 60$ anos), segundo a faixa etária. Caxias do Sul, RS, 2017.

\begin{tabular}{|c|c|c|c|c|}
\hline Macronutriente & Valores de referência (DRI) & 60-70 anos Média $\pm D P$ & $>70$ anos Média $\pm \mathrm{DP}$ & p \\
\hline $\mathrm{HC}(\%)$ & $50-60$ & $62,52 \pm 5,82$ & $60,44 \pm 7,83$ & 0,554 \\
\hline PTN (\%) & $18-20$ & $15,65 \pm 3,21$ & $18,74 \pm 9,88$ & 0,172 \\
\hline LIP (\%) & $15-30$ & $21,90 \pm 5,30$ & $22,19 \pm 8,39$ & 0,408 \\
\hline Fibras (g) & 30 & $17,11 \pm 9,62$ & $17,29 \pm 10,63$ & 0,492 \\
\hline Micronutrientes & Valores de referência (DRI) & 60-70 anos Média $\pm D P$ & $>70$ anos Média $\pm D P$ & p \\
\hline Sódio (mg) & 1,3 & $1115,05 \pm 577,49$ & $1810,2 \pm 1073,88$ & 0,111 \\
\hline Cálcio (mg) & 1200 & $342,4 \pm 190,91$ & $371,84 \pm 143,69$ & 0,75 \\
\hline Ferro (mg) & 8 & $9,89 \pm 3,46$ & $12,21 \pm 6,63$ & 0,37 \\
\hline Vitamina A $(\mu \mathrm{g})$ & 900 & $455,55 \pm 781,43$ & $293,83 \pm 167,12$ & 0,562 \\
\hline Vitamina B6 (mg) & 1,7 & $0,64 \pm 0,21$ & $1,31 \pm 1,71$ & 0,492 \\
\hline Vitamina B12 $(\mu \mathrm{g})$ & 2,4 & $0,79 \pm 0,73$ & $1,84 \pm 1,86$ & 0,093 \\
\hline Vitamina C (mg) & 90 & $119,43 \pm 76,43$ & $117,89 \pm 39,82$ & 0,965 \\
\hline
\end{tabular}

DRI: Dietary Recomended Intakes. 
por Brandão et al. ${ }^{17}$ em que 40 idosos atendidos em um ambulatório de nutrição de um hospital universitário, apresentaram quantidade consumida de fibras acima de 22,0g. O consumo de fibras alimentares está relacionado à constipação intestinal, condição que pode provocar outras doenças através da cronicidade dos sintomas, podendo interferir negativamente na qualidade de vida do indivíduo, sendo que a população residente da zona rural tem menor prevalência de desenvolvimento desta condição devido ao fácil acesso a alimentos como frutas e verduras ${ }^{18,19}$. O consumo isolado de fibras não é suficiente para prevenir a constipação, é necessário uma série de hábitos como adequada ingestão hídrica, de fibras e prática de atividades físicas ${ }^{20}$.

Quando analisada a ingestão de micronutrientes, encontrou-se valores dentro do recomendado pela DRI para o consumo de sódio. O consumo de sal pela população brasileira é maior que o limite máximo de ingestão recomendado pela Organização Mundial da Saúde ${ }^{21,22}$, o que afeta diretamente a saúde do consumidor, visto que a forma de consumo mais utilizada é através do cloreto de sódio, sendo um produto de fácil acesso, usado no tempero de alimentos, seu uso excessivo pode aumentar o risco de doenças cardíacas ${ }^{8,23}$. Durante o envelhecimento são notadas mudanças fisiológicas que afetam a percepção dos sabores, fator que leva a pessoa idosa a adicionar mais sal ao alimento ${ }^{4}$.

A média de cálcio consumida pelos idosos ficou bem abaixo das quantidades recomendadas, embora não significativo, sendo prováveis motivos, o elevado custo dos produtos alimentícios fonte de cálcio, os hábitos culturais e alimentares ${ }^{24}$. Resultado este semelhante aos encontrado por Silva et al. ${ }^{25}$ que avaliaram o consumo de vitaminas e minerais na dieta de 61 idosos encaminhados a um ambulatório de nutrição clínica de um Hospital de Cardiologia de São Paulo, e observaram baixa ingestão do mineral em ambos os gêneros, sendo a ingestão de $371,72 \mathrm{mg}$ para homens e $381,97 \mathrm{mg}$ para mulheres. Resultados obtidos pela Pesquisa de Orçamentos Familiares (2008-2009) ${ }^{26}$, revelam que o cálcio é um dos nutrientes com maior inadequação de consumo pelos idosos. Segundo a Sociedade Brasileira de Reumatologia ${ }^{27} \mathrm{o}$ cálcio é fundamental para na formação óssea e na prevenção da osteoporose, sendo a idade superior a 60 anos e a baixa ingestão do mineral, fatores de risco para o desenvolvimento de tal comorbidade.

Neste estudo observou-se que o consumo de ferro pelos idosos participantes ficou de acordo com o recomendado, dado que difere do estudo realizado por Venturini et al. ${ }^{5}$ em que os idosos entre 60-69 anos possuíam maior consumo do mineral. Segundo Rosa et al..$^{28}$ as principais fontes de ferro na alimentação de idosos são o feijão e as carnes bovinas. Esse resultado pode ser explicado pelo alto consumo de carne pela população do sul do país, sendo consumida em média de uma a três vezes por semana ${ }^{29}$.
Em relação a vitamina A, o baixo consumo foi constatado também no estudo de Silva et al. ${ }^{25}$ com 61 idosos pacientes do ambulatório de nutrição de um Hospital de Cardiologia de São Paulo, em que a ingestão média da vitamina foi de $714,11 \mathrm{mcg}$ para o gênero masculino e $486,06 \mathrm{mcg}$ para o gênero feminino. Mesmo a população brasileira consumindo carnes frequentemente, a qual é alimento fonte dessa vitamina, apresenta baixo consumo de frutas e hortaliças, fonte de carotenoides precursores da vitamina $\mathrm{A}$, o que pode explicar a inadequação ${ }^{30}$.

O processo de envelhecimento torna o idoso susceptível a deficiência de vitaminas do complexo $\mathrm{B}$, fato que pode refletir em parâmetros hematológicos, cardiovasculares e no tecido ósseo ${ }^{31}$. Vinte e seis por cento dos idosos que participaram do estudo de Favero et al. $^{32}$ apresentaram deficiência de cobalamina, corroborando com os achados do presente estudo. Assim como a vitamina B12, o baixo consumo da vitamina B6 também foi encontrado no estudo de Lopes et al. ${ }^{33}$ com 550 adultos e idosos, em que $94 \%$ idosos apresentaram consumo abaixo do adequado.

No estudo de Mendes et al. ${ }^{34}$ avaliaram o consumo alimentar de 17 idosos acamados do município de Batatais-SP, o consumo de cálcio e vitamina B12 ficaram abaixo das recomendações estabelecidas pela DRI e o consumo de ferro estava acima da quantidade recomendada. Porém resultados opostos ao presente estudo foram encontrados para a avaliação dos macronutrientes carboidrato e proteína, visto que a ingestão estava adequada às recomendações.

A vitamina $\mathrm{C}$ esteve dentro das recomendações estabelecidas para idosos, assim como no estudo de Berkenbrock et al. ${ }^{35} \mathrm{com}$ 178 idosos de Florianópolis - Santa Catarina, em que somente as vitaminas $\mathrm{C}$ e A tiveram o consumo adequado, principalmente por idosas do gênero feminino. Esse resultado pode ser explicado pelo consumo de frutas, fontes da vitamina, a qual desempenha papel fundamental na síntese de colágeno e de neurotransmissores, atua como antioxidante contribui para a absorção de ferro, participa na manutenção da pele, gengivas e vasos sanguíneos ${ }^{36}$.

As limitações do presente estudo foram o baixo número de participantes e o método R24h utilizado para avaliar o consumo alimentar dos idosos o qual depende da memória dos entrevistados.

Conclui-se que, os resultados encontrados demonstraram que apesar da grande oferta de alimentos in natura, representados principalmente por frutas, legumes, verduras e leguminosas, que os idosos residentes da zona rural possuem, o consumo desses não é suficiente para atender as recomendações estabelecidas. $\mathrm{O}$ consumo de carboidrato ficou acima das recomendações, que pode ser explicado pela alteração na percepção do paladar aumentada ao doce, diferentemente das fibras que não atingiram o consumo mínimo estabelecido. Os idosos apresentaram baixo consumo de lipídios, cálcio, vitamina A, B6 e B12. O consumo de proteínas, vitamina $\mathrm{C}$, sódio e ferro atingiram as recomendações. 


\section{REFERÊNCIAS}

1. Neumann L, Schauren BC, Adami FS. Sensibilidade gustativa de adultos idosos. Rev Bras Geriatr Gerontol. 2016;19(5):797-808. http://dx.doi.org/10.1590/1809-98232016019.150218

2. Heitor SFD, Rodrigues LR, Tavares DMS. Fatores associados às complicações metabólicas e alimentação em idosos da zona rural. Ciênc Saúde Coletiva. 2016;21(11):3357-66. http://dx.doi.org/10.1590/1413-812320152111.11592015

3. Santos TF, Delani TCO. Impacto da deficiência nutricional na saúde de idosos. Rev Uningá Rev. 2015;21(1):50-4.

4. Brasil. Ministério da Saúde. Alimentação saudável para a pessoa idosa. Um manual para profissionais de saúde. Brasília: Ministério da Saúde; 2009

5. Venturini $C D$, Engroff $P$, Sgnaolin $\mathrm{V}$, Kik RME, Morrone FB, Silva Filho IG, et al. Ingestão inadequada de nutrientes na população de idosos no Brasil: Inquérito Nacional de Alimentação 20082009. Rev Saúde Pública. 2013;47(1 Supl):2225-305 http://dx.doi.org/10.1590/1413-812320152012.01432015

6. Fisberg RM, Marchioni DML, Castro MA, Junior EV, Araújo MC, Bezerra IN, et al. Avaliação nutricional de idosos: desafios da atualidade. Rev. Bras. Geriatr. Gerontol. 2015;18(3):643-50. http://www.scielo.br/pdf/rsp/v47s1/08.pdf

7. Souza AM, Pereira RA, Yokoo EM, Levy RB, Sichieri R. Alimentos mais consumidos no Brasil: Inquérito Nacional de Alimentação 2008-2009. Rev Saúde Pública. 2013;47(1 Supl):1905-95. http://www.scielo.br/pdf/rsp/v47s1/05.pdf

8. Brasil. Ministério da Saúde. Guia alimentar para a população brasileira. $2^{a}$ ed. Brasília: ministério da Saúde; 2014

9. Assumpção D, Domene SMA, Fisberg RM, Barros MBA. Qualidade da dieta e fatores associados entre idosos: estudo de base populacional em Campinas, São Paulo, Brasil. Cad Saúde Pública. 2014;30(8):1680-94.

http://dx.doi.org/10.1590/0102-311X00009113

10. Louzada MLC, Martins APB, Canella DS, Baraldi LG, Levy RB, Claro RM, et al. Impacto de alimentos ultraprocessados sobre o teor de micronutrientes da dieta no Brasil. Rev Saúde Pública. 2015;49:45.

http://dx.doi.org/10.1590/S0034-8910.2015049006211

11. Kuipers RS, Graaf DJ, Luxwolda MF, Muskiet MHA, DijckBrouwer DAJ, Muskiet FAJ. Saturated fat, carbohydrates and cardiovascular disease. J Med. 2011;69(9)372-8.

12. Schmaltz RMLC. Avaliação do consumo alimentar de idosos institucionalizados da cidade de Paracatu, MG. Rev Augustus. 2011;16(32):21-7.

13. Vaz TL, Tagliapietra BL, Schuch NJ, Blasi TC, Margutti KMM. Consumo de proteínas e sua relação com a sarcopenia em idosos. Disciplinarum Sci. 2016;17(1):41-51.

14. Kucera MR, Sivieiro J, Bonatto S. Consumo de lipídeos e estado nutricional de idosos participantes do projeto NUTENV da Universidade de Caxias do Sul. Rev Bras Ciênc Envelhecimento Hum. 2012;9(3):426-38.

http://dx.doi.org/10.5335/rbceh.2012.2811

15. Previdelli AN, GoulartRMM, Aquino RC. Balanço de macronutrientes na dieta de idosos brasileiros: análises da Pesquisa Nacional de Alimentação 2008-2009. Rev Bras Epidemiol. 2017;20(1):70-80. http://dx.doi.org/10.1590/1980-5497201700010006

16. Brasil. Brasil. Ministério da Saúde. Secretaria de Vigilância à Saúde. Secretaria de Atenção à Saúde. Diretrizes e recomendações para o cuidado integral de doenças crônicas não-transmissíveis: promoção da saúde, vigilância, prevenção e assistência. Vol.8. Brasília: Ministério da Saúde; 2008.

17. Brandão JM, Fernandes CS, Barroso SG, Rocha GS. Associação do consumo de fibras e risco cardiovascular em pacientes idosos. Int J Cardiovasc Sci. 2015;28(6):464-71. http://dx.doi.org/10.5935/2359-4802.20150066

18. Klaus JH, Nardin V, Paludo J, Scherer F, Dal Bosco. Prevalência e fatores associados à constipação intestinal em idosos residentes em instituições de longa permanência. Rev Bras Geriatr Gerontol. 2015;18(4):835-43.

http://dx.doi.org/10.1590/1809-9823.2015.13175

19. Heitor SFD, Rodrigues LR, Dias FA, Martins NPF, Tavares DMS. Fatores associados à constipação intestinal em idosos residentes na zona rural. Rev Eletr Enf. 2013;15(4):948-55. http://dx.doi.org/10.5216/ree.v15i4.20546

20. Silva KC, Nobre LN, Vicente SECF, Moreira LL, Lessa AC, Lamounier JA. Influência do índice glicêmico da dieta sobre o risco de sobrepeso e adiposidade na infância. Rev Paul Pediatr. 2016;34(3):293-300.

http://dx.doi.org/10.1016/j.rppede.2015.12.009

21. Sarno F, Claro RM, Levy RB, Bandoni DH, Monteiro CA. Estimativa de consumo de sódio pela população brasileira, 2008-2009. Rev Saúde Pública. 2013;47(3):571-8. http://dx.doi.org/10.1590/S0034-8910.2013047004418

22. Porto AS, Pereira TSS, Molina MCB. Consumo de sódio e potássio por diferentes métodos de avaliação: uma revisão em estudos populacionais. Rev Bras Pesq Saúde. 2014;16(3):131-9. https://doi.org/10.21722/rbps.v16i3.10160

23. Brasil. Ministério da Saúde. Vigilância de fatores de risco e proteção para doenças crônicas por inquérito telefônico Vigitel. Disponível em: http://tabnet.datasus.gov.br/cgi/vigitel/vigteldescr. htm Acesso em: 05 dez. 2017.

24. Pereira GAP, Genaro PS, Pinheiro MM, Szejnfeld VL, Martini LA. Cálcio dietético: estratégias para otimizar o consumo. Rev Bras Reumatol. 2009;49(2):164-80. http://dx.doi.org/10.1590/S0482-50042009000200008

25. Silva BFC, Kovacs C, Magnoli D, Romualdo M. Hábito alimentar e consumo de vitaminas e minerais em idosos. Rev Bras Nutr Clin. 2013;2(3):209-13

26. Instituto Brasileiro de Geografia e Estatística (IBGE). Pesquisa de orçamentos familiares 2008-2009. Análise do consumo alimentar pessoal no Brasil. Rio de Janeiro: IBGE; 2011.

27. Sociedade Brasileira de Reumatologia. Osteoporose: cartilha para pacientes. Sociedade Brasileira de Reumatologia. Comissão de Doenças Osteometabólicas e Osteoporose. 2011.

28. Rosa M, Cantarelli L, Colpo E. Consumo de alimentos com propriedades antioxidantes por idosos institucionalizados. Sci Med. 2014;24(2):116-22.

29. Schneider BC, Duro SMS, Assunção MCF. Consumo de carnes por adultos do sul do Brasil: um estudo de base populacional. Ciênc Saúde Coletiva. 2014;19(8):3583-92. http://dx.doi.org/10.1590/1413-81232014198.11702013

30. Tureck C, Locateli G, Corrêa VG, Koehnlein EA. Avaliação da ingestão de nutrientes antioxidantes pela população brasileira e sua relação com o estado nutricional. Rev Bras Epidemiol. 2017;20(1):30-42. http://dx.doi.org/10.1590/1980-5497201700010003 
31. Coussirat C, Batista C, Schneider RH, Resende TH, Schwanke CHA. Vitaminas B12, B6, B9 e homocisteína e sua relação com a massa óssea em idosos. Rev Bras Geriatr Gerontol. 2012;15(3):577-85 http://dx.doi.org/10.1590/S1809-98232012000300018

32. Favero MT, Griep R, Sulzbacher G. Incidência de idosos com deficiência de vitamina B12 em serviço geriátrico do município de Cascavel-PR - Um estudo transversal. Rev Thêma Scientia. 2015;5:(2E):87-93.

33. Lopes ACS, Caiaffa WT, Sichieri R, Mingoti SA, Lima-Costa MF. Consumo de nutrientes em adultos e idosos em estudo de base populacional: Projeto Bambuí. Cad Saúde Pública. 2005;21(4):1201-9. http://dx.doi.org/10.1590/S0102-311X2005000400022
34. Mendes HS, Toledo GCG. Avaliação do consumo alimentar de idosos acamados pertencentes a duas estratégias de saúde da família em Batatais SP. Saúde, Batatais. 2016;5(1):57-71.

35. Berkenbrock EP, Navarro AC. Consumo alimentar de idosos praticantes de atividade física do município de Florianópolis-SC: Uma abordagem em micronutrientes. Rev Bras Nutr Esportiva. 2011;5(26):145-52

36. Secafim MV, Previdelli AN, Marques KM, Ferreira MPN, Freitas TI, Goulart RMM, et al. Avaliação do consumo de frutas por idosos de São Caetano do Sul, São Paulo, Brasil. Geriatr Gerontol Aging. 2016;10(2):49-56

http://dx.doi.org/10.5327/Z2447-211520161021 